RICYDE. Revista Internacional de Ciencias del Deporte doi: $10.5232 /$ ricyde

Rev. int. cienc. deporte

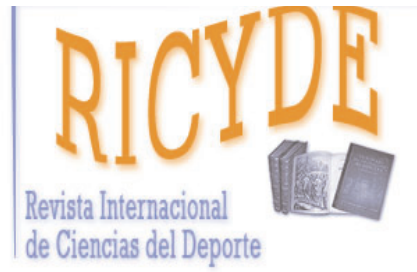

RICYDE. Revista internacional de ciencias del deporte VOLUMEN XI - AÑO XI

Páginas: $116-128$ ISSN : $1885-3137$ No 40 - Abril - 2015

\title{
Influencia de la densidad de jugadores sobre la frecuencia cardíaca y respuestas técnicas en jóvenes jugadores de fútbol \\ Influence of the density of players on their heart rate and its technical implica- tions on young football players
}

\author{
Ricardo Febré ${ }^{1}$, Luis Javier Chirosa 2 , David Casamichana ${ }^{3}$, Ignacio Chirosa ${ }^{4}$, \\ Ignacio Martín-Tamayo5, Carlos Pablos 6
}

1.Instituto de Investigación CCAFyD. Universidad Católica de Valencia

2. Departamento de Educación Física y deportiva. Facultad de Ciencias del deporte. Universidad de Granada

3.Universidad Europea del Atlántico. Santader, Cantábria

4.Departamento de Educación Física y deportiva. Facultad de Ciencias del deporte. Universidad de Granada

5.Departamento de Metodología de Ciencias del comportamiento. Universidad de Granada

6.Instituto de Investigación CCAFyD. Universidad Católica de Valencia

\section{Resumen}

El propósito de este trabajo fue conocer la repercusión sobre los aspectos fisiológicos y técnicos de tres situaciones diferentes de juegos en espacios reducidos (JR) en jóvenes jugadores de fútbol. Las diferentes situaciones estudiadas fueron 3 vs 3 , 4 vs 4 y 5 vs 5 en un espacio de 30×30, donde participaron 10 jugadores jóvenes de fútbol varones (edad $9.3 \pm 0.7$ años; altura $138.5 \pm$ $10.5 \mathrm{~cm}$ y peso de $41.9 \pm 6 \mathrm{~kg}$ y una experiencia de $2.2 \pm 1.4$ años). Las acciones técnicas se cuantificaron a partir de las grabaciones en vídeo y la respuesta fisiológica fue medida a través de la frecuencia cardíaca $\left(\% \mathrm{Fc}_{\mathrm{m}}\right.$ ed y \% $\mathrm{Fc}_{\mathrm{máx}}$ ) y de la percepción subjetiva del esfuerzo (PSE). Los resultados del análisis de varianza (ANOVA) reflejan diferencias significativas en las siguientes variables: pases buenos, pases malos, $\% \mathrm{Fc}_{\text {med }} \% \mathrm{Fc}_{\text {máx }}$, tiempo entre $70-79 \%$ y $>90 \% \mathrm{Fc}_{\text {máx }}$ y PSE. Los resultados encontrados ponen de relieve que la manipulación de la densidad en las tareas tiene efectos a diferentes niveles y por tanto debe ser tenido en cuenta por parte de los técnicos deportivos que trabajan con jugadores jóvenes a la hora de diseñar tareas de entrenamiento. La conclusión principal es que la utilización del formato 3 vs 3 parece ser más demandante tanto a nivel técnico como cardíaco.

Palabras clave: Fútbol; juegos en espacios reducidos; técnica; entrenamiento aeróbico; jóvenes jugadores; esfuerzo percibido y frecuencia cardíaca.

\begin{abstract}
The purpose of this research was to find out the impact of the physiological and technical aspects of three different situations of small-sided games (SSG) on young football players. The different situations considered were 3 vs 3 , 4 vs 4 and 5 vs 5 in a space of $30 \times 30 \mathrm{~m}$, implemented by 10 young male football players (age $9.3 \pm 0.7$; height $138.5 \pm 10.5 \mathrm{~cm}$, weight $41.9 \pm 6 \mathrm{~kg}$ and $2.2 \pm 1.4$ years' experience). The technical actions were quantified out of video recordings and the physiological performance of the exercise was measured through the heart rate $\left(\% \mathrm{HR}_{\text {med }}\right.$ and $\left.\% \mathrm{HR}_{\text {pico }}\right)$, and the rated of perceived exertion (RPE). The results of variance analysis (ANOVA) show significant differences in the following variables: good passes, bad passes, average HR, maximum HR; time $70-79 \%$ and between $>90 \% \mathrm{HR}_{\text {máx }}$ and PSE. The results point out that the manipulation of the density of the tasks has effects on different levels and must therefore be taken into account by the coaches that work with young players when it comes to designing training tasks. The main conclusion is that the use of the 3 vs 3 format seems more demanding both for technical purposes and aerobic performance.
\end{abstract}

Key words: Football; small-sided games; technics, aerobic training; young players; perceived effort and heart rate.

Correspondencia/correspondence: Ricardo Febré Valero

Instituto de Investigación de Ciencias de la Actividad Física y Deportes. Universidad de Valencia. España

E-mail: rifeva2@hotmail.com 
Febré, R.; Chirosa, L. J.; Casamichana, D.; Chirosa, I.; Marín-Tamallo, I.; Pablos, C. (2015). Influencia de la densidad de jugadores sobre la frecuencia cardíaca y respuestas técnicas en jóvenes jugadores de fútbol. RICYDE. Revista internacional de ciencias del deporte, 40(11), 116-128. http://dx.doi.org/10.5232/ricyde2015.04002

\section{Introducción}

$\mathrm{L}$ a preocupación por la enseñanza de los deportes especialmente en sus etapas de iniciación, ha ocupado desde hace tiempo a profesionales e investigadores de la educación física y deportiva (López y Castejón, 2005). Atrás quedó la etapa de la perfecta ejecución de los llamados "fundamentos básicos" para realizar un juego de alto nivel. En las últimas décadas, se puede apreciar un cambio de metodología hacia propuestas más acordes con las necesidades actuales del juego (Gutiérrez y García-López, 2012)

Tradicionalmente, la mayoría de entrenadores utilizaban ejercicios que se ejecutan sin balón para el desarrollo de ciertas capacidades físicas como la resistencia aeróbica (Little y Williams, 2007). Sin embargo, actualmente se piensa que se pueden desarrollar habilidades técnicas, toma de decisiones y resistencia aeróbica en una misma tarea (Köklü, 2012).

La teoría sobre estos planteamientos alternativos de iniciación deportiva está bien desarrollada actualmente (Gonzalez, García, Contreras, y Sánchez-Mora, 2009). Además de un creciente interés por la evaluación de la efectividad del entrenamiento (Hill-Hass, Rowsell, Dawson, y Coutts, 2009; Little y Williams, 2007). Este interés creciente en las ciencias del deporte ha desarrollado programas de formación que ofrecen a los entrenadores de los deportes de equipo, métodos efectivos para la mejora de la formación y del rendimiento del jugador (Keith, Duarte, Vanda, y Luís, 2013).

No obstante, a pesar de esta tendencia y de la popularidad del fútbol, todavía hay muchas incertidumbres relativas a los requisitos multidimensionales del juego (Abrantes, Nunes, Macas, Leite, y Sampaio, 2012). Esta complejidad se puede abordar mediante ejercicios específicos que tienen como objetivo aumentar la participación de los jugadores y preservar la variabilidad del juego básico (Hill-Hass y col., 2009).

Este proceso de formación está centrado en el uso de juegos en espacios reducidos (JR), situaciones motrices de juegos que son modificados, practicados en dimensiones reducidas del terreno utilizando reglas adaptadas y con la participación de un número menor de jugadores (Sampaio, Lago, Gonçalves, Maças, y Leite, 2013). Para un equipo es importante la capacidad que tienen sus jugadores de cooperar en un área determinada del campo con sus compañeros de equipo (Katis y Kelly, 2009), por lo tanto estas condiciones debieran verse reflejadas en los ejercicios de entrenamiento.

Actualmente existe documentación científica que refuerza la utilización de los JR, también denominados con las siglas anglosajonas de SSG (small-sided games) como medios específicos para desarrollar las cualidades principales del jugador de fútbol como la condición física (Hill-Hass, Coutts, Rowsell, y Dawson, 2008), desarrollo técnico (Casamichana, Castellano y Castagna, 2012; Dellal, Drust y Lago-Peñas, 2012; Abrantes y col., 2012) y táctico de los jugadores (Sampaio y Maças, 2012).

La intensidad del ejercicio en los JR ha sido generalmente evaluada a través de la Frecuencia Cardíaca (FC); la concentración del lactato en sangre y la percepción subjetiva del esfuerzo (PSE) (Hill-Haas, Dawson, Impellizzeri, Couts, 2011). Varios estudios han demostrado que tanto la FC como el consumo de oxígeno (VO2) son indicadores válidos para la medir la intensidad del ejercicio en el fútbol (Brito, Krustrup, y Rebelo, 2012; Dellal, Hill-Haas, LagoPenas, y Chamari, 2011).

Igualmente la PSE se ha convertido en una herramienta útil y fácilmente aplicable (Brito y col., 2012; Hill-Hass y col., 2011), es un método simple, no invasivo y de bajo costo económico para controlar la intensidad del ejercicio. Varios estudios han demostrado que 
Febré, R.; Chirosa, L. J.; Casamichana, D.; Chirosa, I.; Marín-Tamallo, I.; Pablos, C. (2015). Influencia de la densidad de jugadores sobre la frecuencia cardíaca y respuestas técnicas en jóvenes jugadores de fútbol. RICYDE. Revista internacional de ciencias del deporte, 40(11), 116-128. http://dx.doi.org/10.5232/ricyde2015.04002

puede ser un indicador válido en la evaluación de la intensidad del ejercicio así como un indicador global de la intensidad de la sesión (Coutts, Rampinini, Marcora, Castagna, Impellizzeri, 2009).

Los JR se definen como situaciones motrices lúdico-deportivos en las que se incluyen todos los factores que intervienen en el juego real de manera simplificada (Hill-Hass y col., 2011). Durante la práctica de este tipo de situaciones, los jugadores experimentan situaciones muy próximas a la competición, además son tareas que se pueden adaptar al grado de dominio del deportista puesto que la complejidad del juego se puede abordar mediante condiciones facilitadas con el objetivo de reducir las interacciones y aumentar la participación de los jugadores (Hill-Hass y col., 2009; Mayo y Navarro, 2008). Estas formas jugadas, reúnen los rasgos de un duelo colectivo y se presentan como un medio específico que permite trabajar a la vez todos los factores mencionados, introduciendo el balón como medio de trabajo (Mallo y Navarro, 2008), provocando una motivación extra (Hoff y Helgerud, 2004) y una optimización del tiempo de trabajo.

Este tipo de situaciones de entrenamiento son cada vez más utilizadas por los entrenadores en todo tipo de deportes de equipo como rugby (Kennett, Kempton, y Coutts, 2012), baloncesto (Sampaio, Abrantes y Leite, 2009) o balonmano (Fruchart, Pâques y Mullet, 2010) quienes modifican diferentes elementos configuradores de la tarea como puede ser el número de jugadores (Kennett y col., 2012), dimensiones del terreno de juego (Brito y col., 2012; Gabbett, Jenkins y Abernethy, 2012; Kelly y Drust, 2009;), las reglas de juego (Arias, Argudo, y Alonso, 2009), aliento del entrenador (Rampinini y col., 2007); incluso la influencia de la superficie de suelo (césped artificial, arena, asfalto) en los juegos reducidos (Brito y col., 2012). Sin embargo poco se sabe sobre la evolución de este tipo de JR a lo largo de las diferentes categorías de edad, desconociendo como se produce la adaptación del niño a este tipo de entrenamiento en sus diferentes etapas formativas.

A pesar de que en el mundo del fútbol el uso de los JR está bien fundamentado, siendo objeto de investigación con diferentes finalidades, la mayoría de los trabajos han sido realizados en el marco del alto rendimiento, teniendo como protagonistas a jugadores profesionales (Dellal y col., 2012), jugadores amateurs (Brito y col., 2012; Casamichana y Castellano, 2010), jugadores juveniles (Owen, Twist, y Ford, 2004) o jugadores jóvenes de entre 13 y 15 años (Abrantes y col., 2012; Da Silva, Impellizzeri, Natali, Bara-Filho, Silami-García, y Marins, 2011), siendo escasos los estudios que revelen la respuesta ante este tipo de situaciones en el proceso de entrenamiento de jugadores que se encuentran en sus primeras etapas formativas (niños de 9 -11 años, benjamines y/o alevines).

Por tanto, el objetivo de este estudio fue examinar cómo afecta la densidad de jugadores en la respuesta cardíaca y en las diferentes acciones técnico-tácticas utilizadas en diferentes formatos de juegos reducidos ( 3 vs $3 ; 4$ vs $4 ; 5$ vs 5 ) realizados en las mismas dimensiones absolutas del espacio (30x30 m).

\section{Método}

\section{Participantes}

En este estudio participaron 10 jugadores jóvenes de fútbol masculino (edad: $9.3 \pm 0.4$ años; altura $138.5 \pm 10.5 \mathrm{~cm} ; \mathrm{Fc}_{\text {máx }} 200.2 \pm 9 \mathrm{ppm}$; peso: $41.9 \pm 6 \mathrm{~kg}$ y una experiencia de $2.2 \pm 1.4$ años en la práctica federada). Todos los jugadores de un equipo "Benjamín (9-10 años)" de nivel regional, participaron en este estudio a excepción de los porteros que quedaron excluidos. Este trabajo se llevó a cabo en el periodo competitivo de la temporada 2011/2012 durante el mes de mayo. Los participantes tenían una frecuencia de entrenamiento de dos 
Febré, R.; Chirosa, L. J.; Casamichana, D.; Chirosa, I.; Marín-Tamallo, I.; Pablos, C. (2015). Influencia de la densidad de jugadores sobre la frecuencia cardíaca y respuestas técnicas en jóvenes jugadores de fútbol. RICYDE. Revista internacional de ciencias del deporte, 40(11), 116-128. http://dx.doi.org/10.5232/ricyde2015.04002

sesiones semanales más el partido de competición, con 48 horas de separación entre cada una de ellas. Los padres fueron informados de los procedimientos de investigación, requisitos, beneficios y riesgos antes de dar su consentimiento. Los participantes fueron informados acerca de la posibilidad de abandonar el estudio en cualquier momento sin penalización alguna. El estudio fue aprobado por el comité de ética de la Universidad Católica de Valencia.

\section{Instrumentos}

Para conocer la frecuencia cardíaca máxima $\left(\mathrm{Fc}_{\text {máx }}\right)$ de los deportistas se realizó el test de potencia aeróbica máxima Course Navette (Cuadrado-Reyes, Chirosa, Chirosa, MarínTamayo y Aguilar Martinez, 2012). Dicha prueba se llevó a cabo en la primera sesión y los valores obtenidos sirvieron para establecer 4 zonas de intensidad cardiaca: la zona $1(<70 \%$ $\left.\mathrm{Fc}_{\text {máx }}\right)$, la zona $2\left(70-79 \% \mathrm{Fc}_{\text {máx }}\right)$; la zona $3\left(80-90 \% \mathrm{Fc}_{\text {máx }}\right)$ y la zona $4\left(>90 \% \mathrm{Fc}_{\text {máx }}\right)$.

Las respuestas de la FC fueron registradas con una frecuencia de muestreo de $0,2 \mathrm{~Hz}$ en todos los JR realizados utilizando telemetría de corto alcance (Polar Team 2). Las correas de los pulsómetros se ajustaron a la medida del niño y los datos fueron transferidos a un ordenador con el software Polar Team 2. Para el estudio se registró la $\mathrm{Fc}_{\text {máx }}$ alcanzada, la $\mathrm{Fc}_{\mathrm{med}}$ así como el tiempo en el cual los jugadores permanecieron en las diferentes zonas de intensidad cardíaca.

Para cuantificar la percepción subjetiva del esfuerzo (PSE) se utilizó la escala de Borg (6-20) reforzada con un dibujo para ayudar a los jóvenes jugadores en sus respuestas. Una semana antes los jugadores utilizaron esta con el fin de familiarizarse con ella. El día que se pasaban los tests, cada vez que finalizaba un juego reducido y durante los 4 minutos de recuperación pasiva, el jugador tenía disponible una hoja con dicha escala, donde marcaba el grado de fatiga que sentían al finalizar dicha sesión (6-20): nada; muy muy ligero; muy ligero; ligero; moderado; un poco pesado; pesado; muy pesado y extremadamente pesado (Cuadrado y col., 2012).

Para el análisis técnico-táctico se utilizó una cámara fija de vídeo Canon Legria HF R47 que se colocó sobre un trípode en la grada a 10 metros detrás del área de juego y a una altura de 2.5 metros sobre el suelo para captar las acciones de los jugadores (Abrantes y col., 2012). Las categorías analizadas fueron: pases, pases buenos, pases malos, regates, entradas e interceptaciones (tabla 1), siguiendo métodos y descripciones utilizadas en estudios anteriores (Kelly y Drust, 2009). Se consideran pases buenos todos aquellos en los que el balón llega al destinatario y tiene un recorrido menor de 15 metros (Mayo y Navarro, 2008).

Tabla 1. Categorías, acrónimo utilizado, definición y grado de apertura del sistema de categorías utilizado en la herramienta de observación.

\begin{tabular}{|l|l|l|}
\hline Categoría & Acrónimo & Núcleo categorial \\
\hline Pases & PAS & $\begin{array}{l}\text { Acción técnica que consiste en establecer una relación entre dos o mas componentes } \\
\text { de un equipo mediante la transmisión del balón por un toque. }\end{array}$ \\
\hline Pases buenos & PASB & $\begin{array}{l}\text { Cuando el balón es jugado por el poseedor y llega al compañero en condiciones de } \\
\text { controlarlo y ser jugado posteriormente }\end{array}$ \\
\hline Pases malos & PASM & $\begin{array}{l}\text { Cuando el balón es jugado por el poseedor y llega al compañero en condiciones de } \\
\text { controlarlo y ser jugado posteriormente }\end{array}$ \\
\hline Regates & REG & $\begin{array}{l}\text { Acción técnica que nos permite avanzar con el balón, conservándolo y desbordando } \\
\text { al contrario para que no intervenga en el juego. }\end{array}$ \\
\hline Entradas & ENT & $\begin{array}{l}\text { Acción físico-técnica que tiene por objeto ir al encuentro del jugador oponente que } \\
\text { está en posesión de balón, con el fin de impedir que progrese en el terreno de juego. }\end{array}$ \\
\hline Interceptaciones & INT & $\begin{array}{l}\text { Acción técnica defensiva por medio de la cual es modificada la trayectoria del balón } \\
\text { evitando o no el fin perseguido por su lanzador. }\end{array}$ \\
\hline
\end{tabular}


Febré, R.; Chirosa, L. J.; Casamichana, D.; Chirosa, I.; Marín-Tamallo, I.; Pablos, C. (2015). Influencia de la densidad de jugadores sobre la frecuencia cardíaca y respuestas técnicas en jóvenes jugadores de fútbol. RICYDE. Revista internacional de ciencias del deporte, 40(11), 116-128.

\section{Procedimiento}

Todos los JR se llevaron a cabo en el mismo campo de césped artificial y en la misma zona del campo, en un espacio de 30 x 30 m (Jones y Drust, 2007; Owen y col., 2004) constante para los tres JR ( 3 vs $3 ; 4$ vs 4 y 5 vs 5 ). Antes de comenzar con la tarea de JR, los jugadores realizaron un calentamiento que incluyó, carrera a baja intensidad, ejercicios dinámicos y estiramientos. Posteriormente, cada sesión consistió en 3 repeticiones de 4 minutos de duración con 3 minutos de recuperación pasiva entre cada una de ellas, en cada una de estas repeticiones se realizaba una situación de juego reducido, según indica la tabla 2.

Al finalizar cada JR los jugadores, de manera individual, le otorgaron un valor a la intensidad de la repetición realizada, utilizando la escala de Borg (6-20). Se dispusieron varios balones alrededor del terreno de juego con el propósito de maximizar el tiempo de juego efectivo mediante la reposición inmediata de los mismos, reduciendo de esta manera el tiempo de cada una de las interrupciones del juego. Durante los JR, los dos entrenadores alentaron a los jugadores (Rampinini y col., 2007). Todas las sesiones fueron realizadas a la misma hora del día (18:00) con el propósito de evitar la influencia de los ritmos circadianos (Chtourou y Souissi, 2012). Durante la práctica se utilizaron las reglas del fútbol 11 a excepción del fuera de juego. Los jugadores fueron divididos en dos equipos. El orden del tipo de juego (3 vs3, 4 vs $4 ; 5$ vs 5) fue modificado de una semana a otra de manera aleatoria (Tabla 2). Previo al estudio, los participantes desarrollaron dos sesiones de familiarización con los procedimientos e instrumentos.

Tabla 2. Orden utilizado en los JR en cada una de las sesiones

\begin{tabular}{|c|c|c|c|c|}
\hline & Numero de jugadores & Duración & Recuperación & $\begin{array}{c}\text { Espacio interacción } \\
\text { individual }\end{array}$ \\
\hline $1^{\text {a }}$ Semana & $\begin{array}{l}4 \text { vs } 4 \\
3 \text { vs } 3 \\
5 \text { vs } 5\end{array}$ & $4^{\prime}$ cada juego & $4^{\prime}$ entre juegos & $\begin{array}{c}112.5 \\
150 \\
90\end{array}$ \\
\hline $2^{\text {a }}$ Semana & $\begin{array}{l}4 \text { vs } 4 \\
3 \text { vs } 3 \\
5 \text { vs } 5\end{array}$ & $4^{\prime}$ cada juego & $4^{\prime}$ entre juegos & $\begin{array}{c}112.5 \\
150 \\
90\end{array}$ \\
\hline $3^{\text {a }}$ Semana & $\begin{array}{l}5 \text { vs } 5 \\
4 \text { vs } 4 \\
3 \text { vs } 3\end{array}$ & $4^{\prime}$ cada juego & $4^{\prime}$ entre juegos & $\begin{array}{c}90 \\
112.5 \\
150\end{array}$ \\
\hline $4^{\mathrm{a}}$ Semana & $\begin{array}{l}3 \text { vs } 3 \\
4 \text { vs } 4 \\
5 \text { vs } 5\end{array}$ & $4^{\prime}$ cada juego & $4^{\prime}$ entre juegos & $\begin{array}{c}150 \\
112.50 \\
90\end{array}$ \\
\hline
\end{tabular}

Para recoger los datos se utilizó metodología observacional, procedimiento científico que permite estudiar la ocurrencia de comportamientos perceptibles de forma que se registren y cuantifiquen adecuadamente (Anguera, y Hernández-Mendo 2013). Las grabaciones en vídeo fueron reproducidas varias veces para asegurar la validez de los datos y garantizar la fiabilidad de las observaciones técnicas (Dellal y col., 2012).

La anotación de cada secuencia se llevó a cabo a través de un sistema de análisis de notación por dos observadores expertos (Abrantes y col., 2012) y los datos fueron registrados en una hoja Microsof Office Excel 2007 y exportadas al Satistical Package for the Social Sciences (SPSS) Statistics 20. 
Se han desarrollado dos formas cuantitativas básicas para hallar la fiabilidad de los datos observacionales (Castellano, Hernández Mendo, Gómez de Segura, Fontetxa, y Bueno, 2000): a) Coeficientes de concordancia entre dos observadores que, registrando de forma independiente, codifican las conductas mediante un mismo instrumento de observación; b) coeficientes de acuerdo, resueltos mediante la correlación. Además, es posible aplicar la teoría de la generalizabilidad, (Casamichana, Castellano, y Hernández-Mendo, 2014) cuando interesa integrar diferentes fuentes de variación (observadores distintos, diversas ocasiones, varios instrumentos, tipos variados de registro, ocasiones diversas, etc.) en una estructura global (Blanco-Villaseñor, Castellano, Hernández-Mendo, Sánchez-López, y Usabiaga, 2014).

\section{Análisis estadístico}

Para el análisis estadístico se realizó un ANOVA de un factor entre grupos (los 3 formatos de JR estudiados) en el que se tuvieron en cuenta todas las variables de medida: pases, pases buenos, pases malos, regates, entradas e interceptaciones, $\mathrm{Fc}_{\text {med }} \mathrm{Fc}_{\text {máx }}$, tiempo de permanencia en cada una de las zonas de intensidad establecidas y PSE. Se realizaron comparaciones post-hoc de Bonferroni para todas las variables. El software utilizado es el Satistical Package for the Social Sciences (SPSS) Statistics 20 y se aplicó un nivel de significación de 0.05 .

\section{Resultados}

La $\mathrm{Fc}_{\max }$ de los jugadores durante la Course Navette fue de $200.2 \pm 9$ lat $/ \mathrm{min}$ ). En la tabla 3 se representan los valores de la $\mathrm{Fc}_{\text {med }} \mathrm{Fc}_{\text {máx }}$ alcanzados por los jugadores en los diferentes $\mathrm{JR}$, así como sus coeficientes de variación. Los resultados del ANOVA muestran que no se han encontrado diferencias significativas en entradas $\left(F_{2,62}=0.7 ; p=0.4\right)$, interceptaciones $\left(\mathrm{F}_{2,62}=0.5 ; \mathrm{p}=0.5\right.$ y zona entre 80 y $90 \% \mathrm{Fc}_{\max }\left(\mathrm{F}_{2,62}=2.3 ; \mathrm{p}=0.1\right)$.

Tabla 3. Frecuencia cardíaca media $\left(\mathrm{Fc}_{\mathrm{med}}\right)$ y máxima $\left(\mathrm{Fc}_{\max }\right)$ obtenida durante los tres JR

\begin{tabular}{|l|c|c|c|}
\hline \multicolumn{1}{|c|}{ Variables } & 3 vs 3 & 4 vs 4 & 5 vs 5 \\
\hline $\mathrm{Fc}_{\text {max }}$ (lat $\left./ \mathrm{min}\right)$ & $190 \pm 10.8$ & $185.7 \pm 9.5$ & $178.55 \pm 11.5$ \\
\hline $\mathrm{Fc}_{\text {med }}($ lat $/ \mathrm{min})$ & $174.71 \pm 11.0$ & $167.86 \pm 9.2$ & $159 \pm 10.1$ \\
\hline
\end{tabular}

La $\mathrm{Fc}_{\text {máx }}$ de los jugadores en los JR durante el juego de 3 vs 3 registró diferencias significativas $\left(\mathrm{F}_{2,62}=6.4 ; \mathrm{p}=0.003\right)$ con respecto al formato de 5 vs 5 , no sucediendo lo mismo con el formato de 4 vs 4 . Igualmente se han obtenido diferencias significativas con la $\mathrm{Fc}_{\mathrm{med}}$ $\left(\mathrm{F}_{2,62}=12.93 ; \mathrm{p}=0.001\right)$, en la que los valores superiores corresponden al juego de 3 vs 3 , mientras que en el formato de 5 vs 5 se obtienen datos inferiores de manera estadísticamente significativa $\left(\mathrm{F}_{2,62}=7.4 ; \mathrm{p}=0.01\right)$, al igual que entre el 4 vs 4 y el 5 vs 5 (Figura 1$)$. 


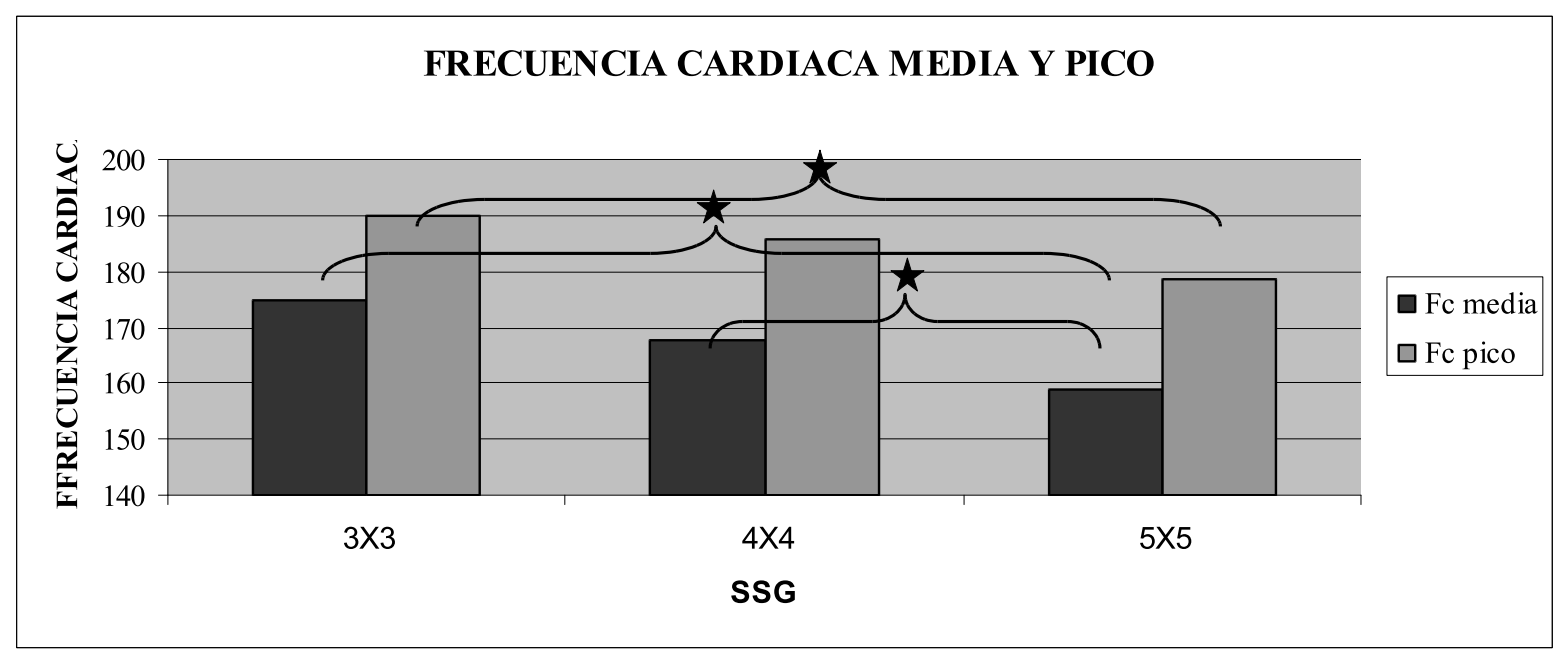

Figura 1. Frecuencia cardíaca media $\left(\mathrm{Fc}_{\mathrm{med}}\right)$ y frecuencia cardíaca pico $\left(\mathrm{Fc}_{\text {pico }}\right)$ en cada uno de los tres juegos reducidos analizados. $*=$ Diferencias significativas $(\mathrm{p}<0.05)$.

En cuanto al tiempo de permanencia en las respectivas zonas de intensidad (Tabla 4) podemos observar cómo se encuentran diferencias significativas en el tiempo invertido en la zona de intensidad $70-79 \% \mathrm{Fc}_{\text {máx }}\left(\mathrm{F}_{2,62}=3.96 ; \mathrm{p}=0.02 ; \eta^{2}=0.09\right)$ y en la zona de intensidad $>90 \%{ }_{\text {máx }}\left(\mathrm{F}_{2,62}=7.98 ; \mathrm{p}=0.001 ; \eta^{2}=0.23\right)$, permaneciendo los jugadores un tiempo significativamente mayor cuando practican situaciones de 3 vs 3 con respecto al 4 vs 4 $(\mathrm{p}=0.001)$.

Tabla 4. Tiempo de permanencia en cada una de las zonas de actividad expresados en segundos.

\begin{tabular}{|l|c|c|c|}
\hline & 3 vs 3 & 4 vs 4 & 5 vs 5 \\
\hline Zona $<70 \%$ & $10.8 \pm 16.4$ & $22 \pm 19.6$ & $33.5 \pm 53.7$ \\
\hline Zona $70-79 \%$ & $20.3 \pm 27.5$ & $22.5 \pm 19.5$ & $39 \pm 2$ \\
\hline Zona $80-90 \%$ & $69.7 \pm 57.9$ & $99.5 \pm 66.7$ & $108.5 \pm 50.6$ \\
\hline Zona $>90 \%$ & $140.3 \pm 74$ & $96.3 \pm 69.7$ & $60.6 \pm 50.5$ \\
\hline
\end{tabular}

En el análisis de la PSE, se encontraron diferencias significativas $\left(F_{2,62}=50.93 ; p=0.001\right.$; $\left.\eta^{2}=0,62\right)$; percibiendo los jugadores como tareas significativamente más intensas los formatos

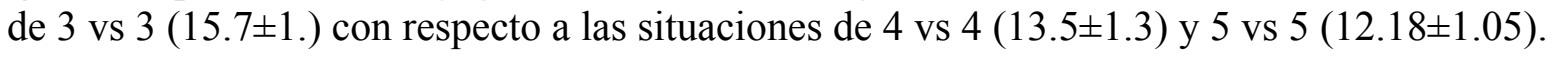

En cuanto al análisis técnico-táctico existen diferencias significativas en el número de entradas $\left(F_{2,62}=0.79 ; p=0.45 ; \eta^{2}=0.02\right)$, pases buenos $\left(F_{2,62}=8.19 ; p=0.001 ; \eta^{2}=0.209\right)$ y pases malos $\left(\mathrm{F}_{2,62}=3.902 ; \mathrm{p}=0.025 ; \eta^{2}=0.112\right)$, sin encontrarse diferencias significativas en la frecuencia de interceptaciones $\left(\mathrm{F}_{2,62}=0.543 ; \mathrm{p}=0.583 ; \eta^{2}=0.017\right)$ entre los diferentes formatos de JR estudiados. Con respecto a los pases buenos, se obtienen diferencias significativas con respecto al 5 vs 5 ( $\mathrm{p}=0.001)$, mientras que no existen diferencias significativas en el resto de los formatos. Con respecto a la frecuencia de pases malos también se registraron diferencias significativas en el formato de 3 vs 3 con respecto al formato de 4 vs 4 ( $p=0.034$ ) (Figura 2). 
Febré, R.; Chirosa, L. J.; Casamichana, D.; Chirosa, I.; Marín-Tamallo, I.; Pablos, C. (2015). Influencia de la densidad de jugadores sobre la frecuencia cardíaca y respuestas técnicas en jóvenes jugadores de fútbol. RICYDE. Revista internacional de ciencias del deporte, 40(11), 116-128.

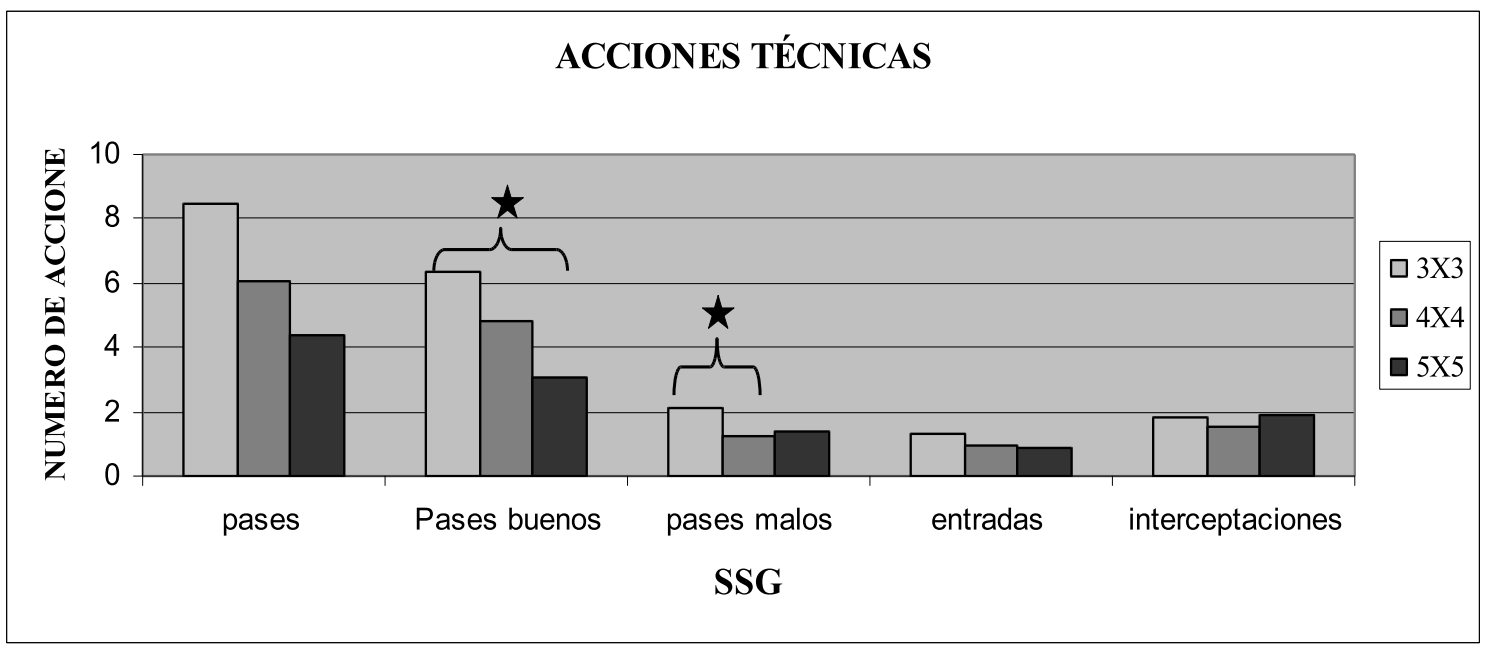

Figura 2. Número de acciones técnico-tácticas realizadas en los tres Juegos Reducidos

\section{Discusión}

El propósito del presente trabajo fue conocer la respuesta de jugadores jóvenes ante JR con diferente densidad de jugadores ( 3 vs 3,4 vs 4 y 5 vs 5) manteniendo constantes las dimensiones absolutas del terreno de juego $(30 \times 30 \mathrm{~m})$. Se trata de un estudio mas, dentro de aquellos que comparan situaciones de JR con pocos jugadores ( 2 vs $2 ; 3$ vs $3 ; 4$ vs 4 o 5 vs 5 ) (Abrantes y col., 2012; Da Silva, Impellizzeri, Natali, De Lima, Bara-Filho, Silami-García, y Marins, 2011; Dellal y col., 2011 ), si bien con la particularidad de que los participantes están en una etapa de iniciación.

Para tal propósito se monitorizó la respuesta cardiovascular que dichos formatos ejercen en los jugadores, la PSE y la frecuencia de diferentes elementos técnico-tácticos (pases buenos, pases malos, entradas, regates e interceptaciones), durante la práctica de situaciones de JR con diferente densidad de jugadores. Para modificar dicha densidad de jugadores por unidad de espacio, se mantienen constantes las dimensiones absolutas del terreno de juego $(30 \times 30)$, modificando el número de jugadores. Los resultados encontrados ponen de relieve que la manipulación de la densidad de jugadores durante las tareas tiene efectos a diferentes niveles y por tanto debe ser tenido en cuenta por parte de los técnicos deportivos que trabajan con jugadores jóvenes a la hora de diseñar tareas de entrenamiento. Sin embargo, los estudios no son consensuados en lo referente a la influencia del tamaño en la respuesta fisiológica de los jugadores.

Los resultados obtenidos en este trabajo vienen a confirmar los obtenidos por otros trabajos en los que participaron jugadores de mayor edad: jugadores de 13-14 años (Da Silva y col., 2011; Katis y Kelly, 2009), jugadores de 15 y 16 años (Abrantes y col. 2012), jugadores de 22 años (Brito y col., 2012) e incluso jugadores profesionales (Little y Williams, 2007), aportando información a un cuerpo de conocimiento cada vez más amplio entorno a los JR (Hill Hass y col., 2010).

Al igual que lo encontrado por otros autores, podemos observar como a medida que se reducen la densidad se aumenta el impacto fisiológico (Abrantes y col., 2012), medido a través de la monitorización de la $\mathrm{FC}$, incrementándose además tanto la participación de los jugadores como la participación exitosa de los mismos. Los resultados indican que la densidad de jugadores es un factor principal que afecta a la frecuencia cardíaca (FC) y a las acciones técnicas. En general, estos estudios han demostrado que en JR con menos jugadores 
Febré, R.; Chirosa, L. J.; Casamichana, D.; Chirosa, I.; Marín-Tamallo, I.; Pablos, C. (2015). Influencia de la densidad de jugadores sobre la frecuencia cardíaca y respuestas técnicas en jóvenes jugadores de fútbol. RICYDE. Revista internacional de ciencias del deporte, 40(11), 116-128.

http://dx.doi.org/10.5232/ricyde2015.04002

se obtiene un ritmo cardíaco mayor que en formatos mas grandes (Hill-Hass y col. 2009); Katis y Kelli, 2009; Owen y col., 2004).

Concretamente los resultados muestran diferencias significativas a nivel de la FC, invirtiendo más tiempo en zonas de alta intensidad cardíaca $\left(>90 \% \quad \mathrm{Fc}_{\text {máx }}\right)$ y alcanzando significativamente valores más elevados en cuanto a $\mathrm{Fc}_{\text {med }} \mathrm{y} \mathrm{Fc}_{\text {máx }}$ cuando la densidad es menor ( 3 vs 3 ) con respecto a los valores obtenidos en el formato de 4 vs 4 y de 5 vs 5 (Casamichana y Castellano, 2010; Mallo y Navarro, 2008; Kelly y Drust, 2009; Little y Williams, 2006; Rampinini y col., 2007).

En lo referente a la PSE, tal y como se ha encontrado en anteriores trabajos, los valores más altos son obtenidos con formatos con un menor número de jugadores (Owen y col., 2004). Este aumento en la PSE también coincide con un aumento en el resto de variables que hacen referencia a la intensidad fisiológica de la tarea, ya que como se ha observado en anteriores trabajos realizados en JR, la correlación entre ambas variables es alta (Casamichana, Castellano, Calleja-González, San Román, y Castagna, 2013). Este aumento en la PSE se justifica en la necesidad de los jugadores de estar en movimiento para crear más oportunidades de juego, ya que la reducción de jugadores reduce el número de soluciones posibles y además aumenta la interacción de cada jugador con el balón, compañeros y adversarios (Owen y col., 2004), lo cual produce un aumento en el gasto energético (HillHaas y col., 2009).

Una elevada frecuencia de acciones técnico-tácticas es un objetivo prioritario de cualquier metodología de enseñanza en el fútbol, por lo que el estudio a este nivel se hace prioritario en el fútbol de formación. En la literatura científica existe cierta controversia en cuanto a cómo influye el número de participantes en la tarea en la frecuencia de acciones técnicas. Algunos autores (Jones y Drust, 2007; Katis y Kelli, 2009) sugieren que con un menor número de jugadores el estímulo de entrenamiento técnico es mas eficaz, puesto que aumenta el número de acciones técnicas, otras investigaciones encuentran un aumento de la frecuencia de las acciones técnicas en los JR cuando se juega con menos jugadores (Owen y col., 2004). Sin embargo, los resultados obtenidos en otros trabajos no permiten identificar diferencias en estas variables (Abrantes y col., 2012; Da Silva y col., 2011). Los resultados de nuestro trabajo no hacen sino confirmar la dificultad de prescribir este tipo de situaciones, ya que no todos los elementos técnico-tácticos se comportan de la misma manera ante la modificación en la densidad de jugadores por unidad de espacio. Mientras que no existen diferencias significativas en algunas variables (regates, entradas, interceptaciones), estas diferencias sí que aparecen cuando la conducta a estudiar son los pases. Tanto la frecuencia de pases buenos como malos es mayor cuando se juega un 3 vs 3 que cuando se juega con mayor número de jugadores y por tanto mayor densidad (4 vs 4 o 5 vs 5 ).

Por tanto, en el formato de 3 vs 3 practicado en un $30 \times 30 \mathrm{~m}$ con respecto al 4 vs 4 y 5 vs 5 en el mismo espacio absoluto, no sólo participan un menor número de jugadores sino que también el espacio relativo a cada participante es mayor, aumentando la intensidad de la tarea y la frecuencia de pases cuando el JR es practicado por jugadores en edades de formación. Parece por tanto que el JR de 3 vs 3 se identifica como una modalidad más próxima al desarrollo de la capacidad aeróbica y a las necesidades propias de un partido de fútbol (Jones y Drust 2007; Kelly y Drust, 2009; Owen y col., 2004). Además incrementa la participación de los jugadores y el número de intervenciones con éxito por jugador, lo cual podría resultar especialmente interesante en el proceso del jugador. 
Febré, R.; Chirosa, L. J.; Casamichana, D.; Chirosa, I.; Marín-Tamallo, I.; Pablos, C. (2015). Influencia de la densidad de jugadores sobre la frecuencia cardíaca y respuestas técnicas en jóvenes jugadores de fútbol. RICYDE. Revista internacional de ciencias del deporte, 40(11), 116-128. http://dx.doi.org/10.5232/ricyde2015.04002

Algunas de las principales limitaciones del trabajo hacen referencia al desconocimiento de la edad madurativa de los participantes, así como la falta de información de la actividad locomotora (Delaserra y col., 2014) y táctico-estratégica (Sampaio y col., 2013) durante este tipo de prácticas, lo cual podría justificar desde una perspectiva más global la utilización de uno $\mathrm{u}$ otro formato en estas categorías de edad tan tempranas. A pesar de ello, estas aportaciones ofrecen nuevos datos a un cuerpo de conocimiento cada vez más amplio sobre JR, habiéndose realizado esta investigación sobre una población específica, jugadores de fútbol base de categoría benjamín, sobre la que no existe demasiada información.

Como conclusión podemos decir que en los JR los jugadores experimentan situaciones muy similares a las de un partido oficial (Owen y col. 2004), de ahí que este método de entrenamiento se haya convertido en un método popular para el entrenamiento de los jugadores de fútbol (Impellizzeri y col. 2006). No obstente, a pesar de la creciente popularidad, la investigación se centra en evaluar las respuestas fisiológicas, tácticas y técnicas de los jugadores cuando se alteran algunos factores como el tamaño del campo, número de jugadores o reglas de juego, pero debido a la falta de coherencia en el diseño de este tipo de juegos (aptitud, edad, nivel, entrenador...) es dificil llegar a conclusiones precisas sobre la influencia de cada uno de estos factores por separado (Aguiar, Botelho, Lago, Maças, y Sampaio, 2012). Unas condiones mas estandarizadas ayudaría a obtener conclusiones mas fiables.

\section{Referencias}

Abrantes, I.; Nunes, I.; Maçãs, M.; Leite, M., \& Sampaio, J. (2012). Effects of the number of players and game type constrainsts on heart rate, rating of perceived exertion, and technical actions of samall-sided soccer games. Journal of Strength and Conditioning Research, 26(4), 976-981. http://dx.doi.org/10.1519/JSC.0b013e31822dd398

Aguiar, M.; Botelho, G.; Lago, C.; Maças, V., \& Sampaio, J. (2012). A review on the effects of soccer small-sided games. Journal of Human Kinetics, 33, 103-113. http://dx.doi.org/10.2478/v10078-012-0049-x

Anguera, M.T., y Hernández-Mendo, A. (2013). La metodología observacional en el ámbito del deporte. E-balonmano.com: Revista de Ciencias del Deporte, 9(3), 135160.

Blanco-Villase-or, A.; Castellano, J.; Hernández-Mendo, A.; Sánchez-López, C., y Usabiaga, O. (2014). Aplicación de la TG en el deporte para el estudio de la fiabilidad, validez y estimación de la muestra. Revista de Psicología del Deporte, 23(1), 131-137.

Brito, J.; Krustrup, P., \& Rebelo, A. (2012). The influence of the playing surface on the exercise intensity of small-sided recreational soccer games. Human Movement Science 31, 946-956.

http://dx.doi.org/10.1016/j.humov.2011.08.011

Casamichana, D.; Castellano, J., \& Castagna, C. (2012). Comparing the physical demands of friendly matches and small-sided games in semiprofessional soccer players. Journal of Strength and Conditioning Research, 326(3), 837-843.

Casamichana, D.; \& Castellano, J. (2010). Time-motion, heart rate, perceptual and motor behaviour demands in small-sides soccer games: Effects of pitch size. Journal of Sports Sciences, 28(14), 1615-1623.

http://dx.doi.org/10.1080/02640414.2010.521168 
Febré, R.; Chirosa, L. J.; Casamichana, D.; Chirosa, I.; Marín-Tamallo, I.; Pablos, C. (2015). Influencia de la densidad de jugadores sobre la frecuencia cardíaca y respuestas técnicas en jóvenes jugadores de fútbol. RICYDE. Revista internacional de ciencias del deporte, 40(11), 116-128.

http://dx.doi.org/10.5232/ricyde2015.04002

Casamichana, D.; Castellano, J., y Hernández-Mendo, A. (2014). La teoría de la generalizabilidad aplicada al estudio del perfil físico durante juegos reducidos con diferente orientación del espacio en fútbol. RICYDE. Revista Internacional de Ciencias del Deporte, 10(37), 194-205.

http://dx.doi.org/10.5232/ricyde2014.03702

Casamichana, D.; Castellano, J.; Calleja-González, J.; San Román, J., \& Castagna, C. (2013). Relationship between indicators of training load in soccer players. Journal of Strength \& Conditioning Research. 27(2), 369-374. http://dx.doi.org/10.1519/JSC.0b013e3182548af1

Castellano, J.; Hernández Mendo, A.; Gómez de Segura, P.; Fontetxa, E., y Bueno, I. (2000). Sistema de codificación y análisis de la calidad del dato en el fútbol de rendimiento. Psicothema, 12(4), 636-641.

Coutts, A.; Rampinini, E.; Marcora, S.; Castagna, C., \& Impellizzeri, F. (2009). Heart rate and blood lactate correlates of perceived exertion during small-sided soccer games. Journal of Science and Medinice in Sport, 12(1), 79-84. http://dx.doi.org/10.1016/j.jsams.2007.08.005

Cuadrado-Reyes, J.; Chirosa Rios, L.J.; Chirosa Rios I.J.; Marín-Tamayo I., y AguilarMartinez, D. (2012). La percepción subjetiva del esfuerzo para el control de la carga de entrenamiento en una temporada en un equipo de balonmano. Revista de Psicología del Deporte, 21, 2, 331-339.

Chtourou, H., \& Souissi, N. (2012). The effect of training at a specific time of day: A review. Journal of Strength and conditioning research, 26(7), 1984-2005. http://dx.doi.org/10.1519/JSC.0b013e31825770a7

Gonzalez, S.; García, L.M.; Contreras, O., y Sánchez-Mora, D. (2009). El concepto de iniciación deportiva en la actualidad. Nuevas Tendencias en Educación Física, Deporte y Recreación, 15(3), 14-20.

Da Silva, C.; Impellizzeri, F.; Natali, A.; De Lima, J.; Bara-Filho, M.; Silami-García, E., \& Marins, J. (2011). Exercise intensity and technical demands of small-sided games in young brazilian soccer players: effect of number of players, maturation, and reliability. Journal of Strength and Conditioning Research, 25(10), 2746-2751. http://dx.doi.org/10.1519/JSC.0b013e31820da061

Dellaserra, CL.; Gao Y., \& Ransdell L. (2014). Use of integrated technology in team sports: a review of opportunities, challenges, and future directions for athletes. The Journal of Strength and Conditioning Research. 28(2), 556-73. http://dx.doi.org/10.1519/JSC.0b013e3182a952fb

Dellal, A.; Drust, B., \& Lago-Penas, C. (2012). Variation of activity demands in smallsided soccer games. International Journal of Sports Medicine, 33, 370-375. http://dx.doi.org/10.1055/s-0031-1295476

Dellal, A.; Hill-Haas, S.; Lago-Penas, C., \& Chamari, K. (2011). Small-sided games in soccer: amateur vs. professional players' physiological responses, physical, and technical activities. Journal of Strength and Conditioning Research, 25(9), 23712381.

http://dx.doi.org/10.1519/JSC.0b013e3181fb4296

Fruchart, E.; Pâques, P., \& Mullet, E. (2010). Decision-making in basketball and handball games: A developmental perspective. Revue Européenne de Psychologie Appliquée/European Review of Applied Psychology, 60(1), 27-34. http://dx.doi.org/10.1016/j.erap.2009.10.003 
Febré, R.; Chirosa, L. J.; Casamichana, D.; Chirosa, I.; Marín-Tamallo, I.; Pablos, C. (2015). Influencia de la densidad de jugadores sobre la frecuencia cardíaca y respuestas técnicas en jóvenes jugadores de fútbol. RICYDE. Revista internacional de ciencias del deporte, 40(11), 116-128.

Gabbett, T.; Jenkins, D., \& Abernethy, B. (2009). Game-based training for improving skill and physical fitness in team sport athletes. International Journal of Sports Science \& Coaching, 4(2), 273-283. http://dx.doi.org/10.1260/174795409788549553

Gutiérrez, D., y García López, L.M. (2012). Evaluación de la toma de decisiones por parte de alumnos de primaria en relación a contextos tácticos. New Approaches In Educational Research, 1, 2254-7339.

Hill-Haas, S.; Coutts, A.; Rowsell, G., \& Dawson, B. (2008). Variability of acute physiological responses and performance profiles of youth soccer players in smallsided games. Journal of Science and Medicine in Sport, 11(5), 487-490. http://dx.doi.org/10.1016/j.jsams.2007.07.006

Hill-Haas, S.; Rowsell, G.; Dawson, B., \& Coutts, A. (2009). Generic versus small-sided game training in soccer. International Journal of Sports Medicine, 30(9), 636. http://dx.doi.org/10.1055/s-0029-1220730

Hill-Haas, S.; Dawson, B.; Impellizzeri, F., \& Coutts, A. (2011). Physiology of small-sided games training in football: a systematic review. Sports Medicine, 41(3), 199-220. http://dx.doi.org/10.2165/11539740-000000000-00000

Hill-Hass, S.; Rowsell, G.; Coutts, A., \& Dawson, D. (2008). The reproducibility of physiological responses and performance profiles of youth soccer players in smallsided games. International Journal of Sports Physiology and Performance, 3(3), 393396.

Hill-Haas, S.; Rowsell, G.; Dawson, B., \& Coutts, A. (2009). Acute physiological responses and time-motion characteristics of two small-sided training regimes in youth soccer players. Journal of Strength and Conditioning, 23(1), 111-115. http://dx.doi.org/10.1519/JSC.0b013e31818efc1a

Hoff, J., \& Helgerud, J. (2004). Endurance and strength training for soccer players: physiological considerations. Sports Medicine, 34(3), 165-180. http://dx.doi.org/10.2165/00007256-200434030-00003

Jones, S., \& Drust, B. (2007). Physiological and technical demands of $4 \vee 4$ and $8 \vee 8$ games in elite youth soccer players. Kinesiology, 39(2), 150-156.

Katis, A., \& Kellis, E. (2009). Effects of small-sided games on physical conditioning and performance in young soccer players. Journal of Sports Science and Medicine, 8, 374-380.

Keith, D.; Duarte A.; Vanda C., \& Luís, V. (2013). How small-sided and conditioned games enhance acquisition of movement and decision-making skills. Exercise \& Sport Sciences Review, 41, 154-161.

http://dx.doi.org/10.1097/JES.0b013e318292f3ec

Kelly, D., \& Drust, B. (2009). The effect of pitch dimensions on heart rate responses and technical demands of small-sided soccer games in elite players. Journal of Science and Medicine in Sport, 12(4), 475-479.

http://dx.doi.org/10.1016/j.jsams.2008.01.010

Kennett, D.; Kempton, T., \& Coutts, A. (2012). Factors affecting exercise intensity in rugby-specific small-sided games. Journal of Strength \& Conditioning Research, 26(8), 2037-2042.

http://dx.doi.org/10.1519/JSC.0b013e31823a3b26

Köklü, $Y(2012)$. A comparison of physilological responses to various intermittent and continuous small-sided games in young soccer players. Journal of Human Kinetics 31,89-96.

http://dx.doi.org/10.2478/v10078-012-0009-5 
Little, T., \& Williams, A. (2007). Measures of. Apunts, 79, (40-48).

Mallo, J., \& Navarro, E. (2008). Physical load imposed on soccer players during smallsided training games. Journal of Sports Medicine and Physical Fitness, 48(2), 166171.

Owen, A.; Twist, C., \& Ford, P. (2004). Small-sided games: the physiological and technical effect of altering pitch size and player numbers. Insight, 7, 50-53.

Rampinini, E.; Impellizzeri, F.; Castagna, C.; Abt, G.; Chamari, K.; Sassi, A., \& Marcora, S.(2007). Factors influencing physiological responses to small-sided soccer games. Journal of Sports Sciences, 25(6), 659-666. http://dx.doi.org/10.1080/02640410600811858

Sampaio, J., \& Maçãs, V. (2012). Measuring tactical behaviour in football. International Journal Sports Medicine, 33(5), 395-401. http://dx.doi.org/10.1055/s-0031-1301320

Sampaio, J.; Abrantes, C., \& Leite, N. (2009). Power, heart rate and perceived exertion responses to $3 \times 3$ and $4 \times 4$ basketball small sided games. Revista de Psicología del Deporte, 18(3), 463-467.

Sampaio, J.; Lago C.; Gonçalves B.; Maçãs V., \& Leite, M. (2013). Effects of pacing, status and unbalance in time motion variables, heart rate and tactical behaviour when playing 5-a-side football small-sided games. Journal of Science and Medicine in Sport, 92-3, 1440-2440. 